\title{
Elevator Drive Control system based on single Chip Microcomputer
}

\author{
Haojun Qin \\ Mechanical Engineering College, WuHan University, WuHan, Hubei
}

\author{
Keywords: MCU; Sine pulse width modulation technology; Elevator drag
}

\begin{abstract}
Today's elevators are increasingly demanding speed, safety, comfort and intelligence, while old-fashioned elevators are struggling to meet today's demands. An elevator drive control scheme based on 51 series chips is designed. The VVVF (variable Voltage Frequency Control) technology is selected as the transmission control mode of the system. The single-chip microcomputer control technology and the SPWM (sinusoidal pulse width modulation) technology are adopted to change the frequency and voltage of the traction motor power supply. The purpose of accurately controlling elevator speed is realized. It has some practicability in the field of retrofit of old elevator and design of new elevator system.
\end{abstract}

\section{Introduction}

In modern life, with the emergence of large numbers of high-rise residential buildings and supermarkets, as an important transportation of vertical transport personnel and goods, transmission equipment, elevators has become more and more indispensable. The promotion of elevator brings a variety of convenience to the city, such as saving a large number of land resources, providing great convenience for traffic, beautifying the city, but also producing a variety of problems. Some old elevators have become normal because of the backwardness of control system, long operation life, and frequent failures in use process. At present, the supervision of elevators in our country is not very perfect, and there is no rigid requirement for the end-of-life of elevators, which has led to a large number of "elderly" elevators that still insist on running with diseases and not to scrap. Many security problems have endangered the lives and property of users, and the pain of these cities is worrying. And the old elevator replacement and transformation has become an urgent hot issue. PLC (Programmable Logic Controller) is used as the control unit in most old elevator reconstruction projects, but the cost of PLC control system is too high, and the real-time and stability are poor. In view of the above background, the author finally selected VVVF (variable Voltage Frequency Control) technology, and designed the system with MCU as the core controller. This design simplifies the hardware circuit of VVVF technology and maximizes the use of SCM resources to improve efficiency.

\section{Summary}

The design system uses 52 series single chip microcomputer as the control core, generates SPWM (sinusoidal pulse width modulation wave) and ideal speed curve in elevator operation, and adopts photoelectric coding disk as the sensor of real-time speed acquisition. The collected speed information is transmitted to the control chip in the form of pulses. The single-chip microcomputer adjusts the speed and state of the elevator by comparing the deviation between the feedback value of the speed sensor and the preset speed value. The speed of elevator is controlled by changing the parameters of SPWM wave. The system design block diagram is shown in figure 1. 


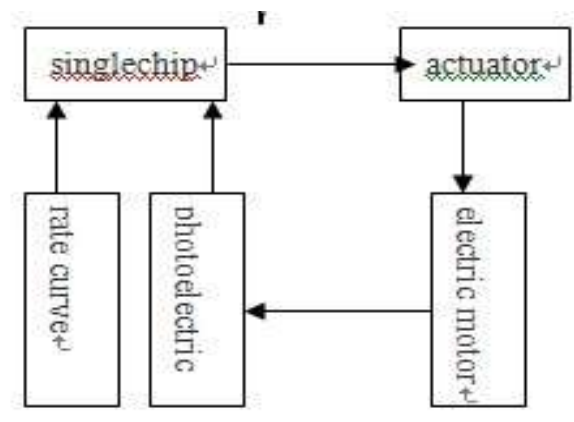

Fig 1 System design block diagram

\section{SPWM Speed Regulation Technology}

SPWM (sinusoidal pulse width modulation) technology is one of the PWM (Pulse Width Modulation) technologies. One of the advantages of using PWM technology is that both the signals from the processor and the signals from the controlled system are transmitted in the form of digital signals. Then do D-A conversion. This advantage is to minimize the interference of noise signals.

One of the advantages of using PWM over analog control is that it is highly resistant to noise. When the analog signal is converted by A / D to a PWM wave, the communication distance can be greatly increased, and the influence of the high frequency square wave can be eliminated by adding a proper filter ( $\mathrm{RC}$ or PWM ) circuit at the receiving end, and then conversing D / A, and restoring the initial analog signal.

At present, PWM technology is widely used in the classification of SPWM technology, and the multiplex PWM technology, which has been recognized by most people, is also a kind of SPWM technology. The performance of this technology in electrical protection is relatively comprehensive and outstanding. The self-contained error detection circuit can detect and display a series of common faults, such as over voltage, over current, equipment open circuit, etc. In peacetime use and maintenance process provided a great deal of convenience.

\section{Generation of a Given Velocity Curve}

On the premise of balancing efficiency and comfort, the generation of elevator running curve has become a research hotspot. At present, there is no consistent standard for the optimal speed curve of elevator operation at home and abroad, but there are relatively uniform principles and empirical constraints.

The most important thing of building passenger elevator system is to meet the needs of people, so it is very important to set up the speed curve according to people's physiological characteristics. The acceleration parameter and acceleration rate of elevator should not be too large or too small. According to experience, the maximum value of acceleration should not exceed $1.5 \mathrm{~m} / \mathrm{s}$, and the maximum rate of change of acceleration should not exceed $1.5 \mathrm{~m} / \mathrm{s}$.

Both adults and children are more sensitive to acceleration changes, the acceleration will suddenly become larger or smaller will lead to increased discomfort in the human body, so there will be more restrictions on the requirements of the velocity curve. What the design velocity curve must do is that the given value of velocity and acceleration should be non-discrete and the value of acceleration change rate should not be abrupt.

In order to ensure the accuracy of the parking position, acceleration and speed of the elevator in the horizontal process, so that they all meet the human body's requirements for comfort, It is necessary to change the shape of the running curve by adjusting the parameters of the given curve during the deceleration of the car in real time.

In the design and generation of the optimal running curve of the elevator, not only the requirements and expectations of the passengers for comfort should be taken into account, but also 
the factors such as safety and stability should be taken into account. The method of limiting the maximum speed of elevator operation is adopted to meet the safety performance index. On this basis, it is necessary to increase the speed of the car in order to meet the initial design requirements of the elevator, and make the elevator run as quickly as possible in the safest possible condition.

The theoretical curve model of the optimal operation of the elevator generated by this design is shown in figure 2.

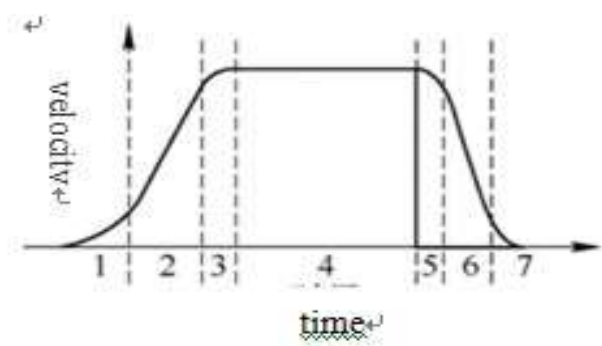

Fig 2 optimal speed curve of elevator operation

\section{Speed regulation}

This design uses SPWM technology, uses photoelectric encoder to collect speed information, compares the given speed curve with closed loop control, and corrects the speed of elevator operation in real time.

\section{Speed given}

Normally, the elevator trajectory can be divided into three processes: starting process (raising speed operation, steady running (constant speed running), and decelerating process (decelerating operation). The frequent operation and switching of the starting and braking actions of the elevator is a challenge to the speed regulation of the motor. If we want to ensure that the elevator is fast, safe and comfortable, it is very important to give the speed. Most of the speed of an old elevator uses an integrator, and the starting process, the deceleration process, is performed by timing (setting the time for starting and starting braking). This is easy to cause the elevator operation efficiency decline. The speed curve of this design is generated and given by the program. The operating parameters of the elevator are determined by comparing and judging the running environment of the elevator, and changing the elevator speed and acceleration. The program is edited by $\mathrm{C}$ language and burned into the single chip microcomputer. The optimal curve is used as the benchmark in the whole running process of the elevator. In the stage of deceleration, the photoelectric coding disk is used as the reference for the flat layer positioning. According to the output signal, the height of the elevator can be determined, and the control unit sends out the signal of deceleration, and the control system automatically discriminates according to the feedback, and sends out the signal of deceleration until it stops running.

\section{Velocity detection}

Most of the speed measurement modules used in the old elevator are based on backward speed measuring elements. For example, the speed measuring generator has some obvious defects, and the speed measurement results are nonlinear. The result is that the dead zone of velocity measurement is not ideal when the speed is low, and the running speed curve is shown in figure 3. So in this design, the photoelectric encoder is used to measure its speed and position. Its communication interface with single chip microcomputer is very simple. It can connect the terminal of output pulse signal directly to the EPA (event processing matrix) of single chip computer without redundant control circuit. You can use a variety of coding methods to perform operations.

Control methods

The best state of the motor in normal operation is to maintain the magnetic flux of each polarity to be stable. The potential energy of each phase of the stator of the motor is expressed as follows: 


$$
E=4.44 f N k_{N} \Phi_{M}
$$

It can be concluded from the upper formula that only the modified $\mathrm{E}$ and $\mathrm{f}$ input values can be adjusted $\Phi_{M}$. In this case, the PWM technology can meet the technical requirements.

The principle of SPWM generation, as shown in figure 4, is actually a new pulse wave, which is generated by comparing the carrier wave (which is a continuous isosceles triangular wave) with the modulated wave (the sine wave is used in this design). The ratio of the instantaneous maximum $U_{m m}$ of the modulated wave to the instantaneous maximum $U_{m}$ of the carrier wave is the modulation system (symbol M). The ratio between the frequency of the triangular wave $f_{r}$ and the frequency of the sinusoidal wave $f_{r}$ is called the carrier ratio (symbol N).

There are many methods to generate SPWM wave. This design chooses a method to generate SPWM wave by application software. The sampling method is the regular sampling method. The regular sampling method determines the duration of the pulse by the absolute value of the distance between the two intersecting points by overlapping the modulated wave with the carrier. The specific process is shown in figure 3.

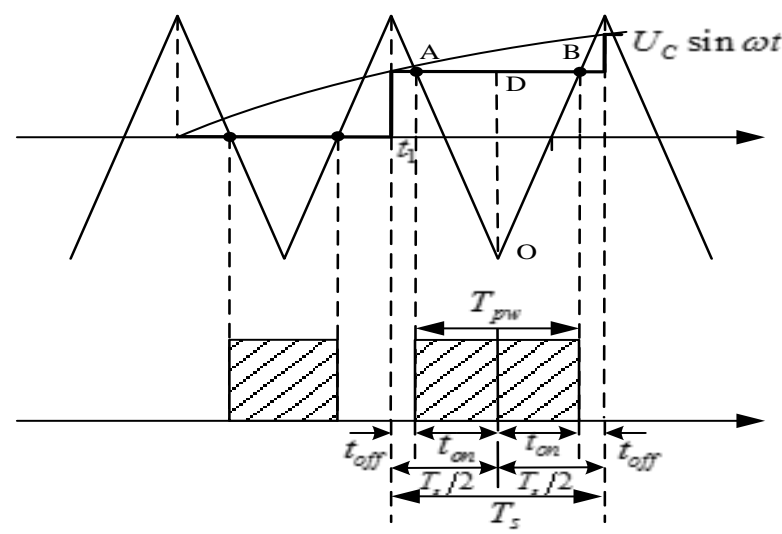

Fig 3 symmetric regular sampling method

In Figure 3, $T_{r}$ is the duration of a carrier cycle, $U_{r}$ is the peak of the carrier, $U_{c} \sin \omega t$ is the modulated wave, and $T_{s}$ is the duration of a sampling. $\omega_{\text {is }}$ the angular frequency of the modulated wave. If $\omega$ is changed, the fundamental frequency of the SPWM pulse train will also change. $t_{1} 、 t_{2}$ is the time point at which the modulated wave intersects with the carrier in two adjacent periods. It is the time point of sampling point (this design adopts vertex sampling). The width of the pulse is:

$$
t_{p w}=\frac{T_{s}}{2}\left(1+M \sin \omega t_{1}\right)=\frac{T_{t}}{2}\left(1+M \sin \omega t_{1}\right)
$$

For the status shown in figure 3, there are:

$$
T_{1}=k T_{t}, k=0,1,2,3 \cdots
$$

From the above formulas, as shown in Fig. 3, the value $t_{\text {off }}$ and $T_{p w}$ of the sampling period at which the point is located can be calculated simply by determining the value of the point $t_{1}$.

The pulse width time ${ }^{T_{p w}}$ of SPWM waveform is calculated as follows:

Assuming that D point is a point of under $U_{r m}$, it intersects the triangle wave at $\mathrm{A}$ and $\mathrm{B}$, and the absolute value between two points is the pulse width time $T_{p w}$ of SPWM wave.

Let the maximum of the triangle wave is $U_{r m}$ the maximum of the sine wave is $U_{r m}$. Regulation system $\boldsymbol{M}=\boldsymbol{U}_{r m} / \boldsymbol{U}_{t m}$

The length values of the two right edges of $\triangle \mathrm{AOD}$ are: 
$|A D|=\mathrm{T}_{2} / 2 \quad|\mathrm{OD}|=\mathrm{U}_{\mathrm{tm}}+\mathrm{U}_{\mathrm{rm}} \sin \omega \mathrm{t}_{\mathrm{d}}$

Further calculation

$|\mathrm{OD}|=\mathrm{U}_{\mathrm{tm}}\left(1+\mathrm{Msin}_{\mathrm{m}} \mathrm{t}_{\mathrm{d}}\right)$

The length values of the two right edges of $\triangle \mathrm{EOF}$ are:

$|\mathrm{EF}|=2 \mathrm{U}_{\mathrm{tm}} \quad|\mathrm{FO}|=\mathrm{T}_{\mathrm{c}} / 2$

That is

$|\mathrm{EF}| /|\mathrm{OD}|=|\mathrm{OD}| /|\mathrm{AD}|$

The pulse width time of SPWM waveform is obtained.

$\mathrm{T}_{2}=\left(1+\mathrm{M} \sin \omega \mathrm{t}_{\mathrm{d}}\right) \mathrm{T}_{\mathrm{c}} / 2$

Gap time:

$\mathrm{T}_{1}=\mathrm{T}_{3}=\left(\mathrm{T}_{\mathrm{c}}-\mathrm{T}_{2}\right) / 2$

Triangular wave period:

$\mathrm{T}_{\mathrm{C}}=\frac{4 \times \text { WG_RELOAD }}{\mathrm{F}_{\mathrm{Xtal}}}$

PWM duty cycle $=\frac{\text { WG_COMP }}{\text { WG_RELOAD }} \times 100 \%$

The symbolic description in the formula:

WG_RELOAD is a 16-bit reload register;

$\mathrm{F}_{\text {xtal }}$-XTAL1 is Crystal frequency on pin

WG_COMP is phase comparator

The value of WG_RELOAD is determined according to the given value of the speed running curve, the value of the tuning system $\mathrm{M}$ is given according to the real time velocity value returned by the photoelectric encoder, and the value sinct $_{\mathrm{a}}$ determined by the characteristic of the sinusoidal function curve. The frequency of the SPWM wave can be calculated by adding it to the above formula.

\section{System control}

The design adopts the execution of the program to control the track of the car, uses the photoelectric encoder to calculate the real-time running speed, and then feedback the relevant value of the encoder to the single-chip microcomputer to process it. It is compared with the preset value on the preset velocity curve, and the contrasted deviation is transmitted to WFG in time for reference to adjust the parameters of SPWM wave. According to the above calculation process, it can be found that the frequency of the SPWM wave and the period of the carrier wave ${ }^{T_{c}}$ are significant. The carrier waves associated with the write value, so we can change the frequency of the triangle wave by taking the method of changing the loading value. The advantage of this method is that the relevant parameters can be changed by changing the duty cycle of SPWM wave, and achieve the desired speed regulation purpose.

\section{Running and debugging}

In the process of actual running and testing, it takes up a lot of memory space to ge nerate the speed curve, but in the whole control system, there are many other tasks besides the speed curve that need to be processed in parallel, such as communication function, etc. Direct operation will increase the repeatability of single-chip computer, decrease the efficiency and weaken the stability of 
single-chip computer. In order to consider this aspect, another processing method, that is, establishing state machine method is adopted in the calculation program of velocity curve. By using this method, the optimal velocity curve is divided into several small sections. In a particular case, the single chip processor only processes the relevant information of the corresponding paragraph, and calculates the velocity curve of the paragraph. In order to reduce the calculation amount and enhance the stability of single chip microcomputer, each paragraph is switched by the sign quantity.

In the process of debugging, curve deformation and error occurred, and the problems were found through many experiments and related data. After the output SPWM wave is converted to analog quantity by D / A, it is necessary to keep the sampling interval regular when it is transmitted to the converter, and to unify the sampling period in each individual velocity curve. After many revisions, a method of program compensation was finally adopted. In the control process, a precise timing method was adopted to transfer the transfer program of the analog speed curve generated after the D I A conversion to the program that interrupts the timing service. The advantage is that the error in time can be greatly reduced, and this error is absolute error, not only can be calculated by calculation, but also the error value will not change, so the effect of this error can be eliminated by program compensation.

Finally, the fitting condition of the velocity curve and the optimal velocity given curve is good in the course of the actual operation, and the system delay is eliminated after adopting the program compensation method. The comparison between the measured running curve of deceleration section and the given velocity curve is shown in figure 4 . The fitting effect is good and can meet the requirements of the design.

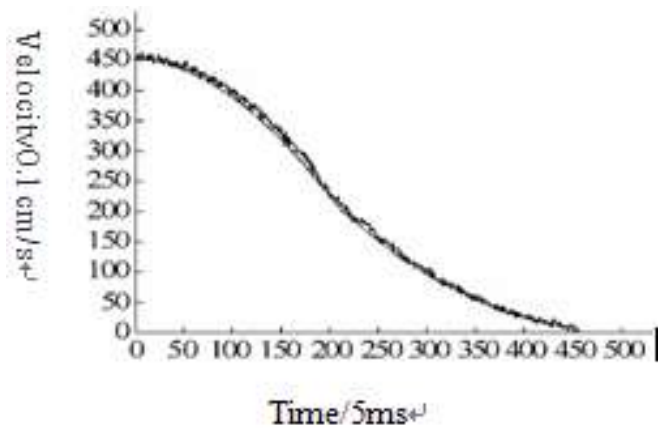

Fig 4 the relationship between measured curve and given curve

\section{Conclusion}

By comparison, the design has the advantages of lower cost, smaller and simpler system structure, more convenient and flexible control and operation, more safe, stable and reliable. This design gives full play to the performance of single chip computer and is innovative in speed and SPWM wave generation.

\section{References}

[1] Dafang Tang, Weng Shaojie, Li Kehong, et al. Strengthen the management of old elevators and promote the transformation of old elevators [J]. China Special equipment Safety 6: 185-187.

[2] Zhongyang Wang. Discussion on the causes of the Hidden trouble of Elevator and the Countermeasures of Supervision and Inspection [J]. Mechatronic information 15: 65-67.

[3] Xiaobing Sun. Problems and Countermeasures of Elevator Supervision system in China [D]. Shijiazhuang: school of Law and Public Administration, Hebei normal University. 2016.

[4] Sijing Tao. Study on elevator safety management [D]. Tianjin: school of Precision instrument and Optoelectronics Engineering, Tianjin University. 2012.

[5] Yanfeng Kong. Analysis of Electrical problems and Inspection in Elevator braking system [J]. Science and Technology Innovation and Application 10: 162-165.

[6] Guohua Chen, Wenjie Cai etc. Elevator risk Pre-assessment method based on large sample Test data [J]. Chinese Journal of Safety Sciences, 2015: 56-60. 
[7] Boshi Chen. Electric drive automatic control system: motion control system [M]. Beijing: mechanical Industry Press, 2003.

[8] Peng L, Xiaoping Z. Social Stratification and Cooperative Behavior in Spatial Prisoners' Dilemma Games [J]. PLOS ONE, 2015, 10(7): e0131005. 